\title{
Machine Learning aided Fiber-Optical System for Liver Cancer Diagnosis in Minimally Invasive Surgical Interventions
}

\author{
E. Zherebtsov ${ }^{1,2}$, M. Zajnulina ${ }^{3}$, K. Kandurova ${ }^{2}$, V. Dremin ${ }^{2,3}$, A. Mamoshin ${ }^{2,4}$, E. Potapova ${ }^{2}$, S. Sokolovski ${ }^{3}$, \\ A. Dunaev ${ }^{1}$, and E.U. Rafailov ${ }^{3}$ \\ ${ }^{1}$ University of Oulu, Optoelectronics and Measurement Techniques Unit, Oulu, Finland \\ ${ }^{2}$ Research and Development Center of Biomedical Photonics, Orel State University, Orel, Russia \\ ${ }^{3}$ Aston Institute of Photonic Technologies, Aston University, Birmingham, UK \\ ${ }^{4}$ Orel Regional Clinical Hospital, 302028 Orel, Russia \\ e-mail: evgenii.zherebtsov@oulu.fi
}

\begin{abstract}
A flexible fibre optical probe is implemented to record the parameters of the endogenous fluorescence during minimally invasive interventions in patients with cancers of hepatoduodenal area. Using machine learning techniques, the obtained spectra are classified to indicate cancerous or healthy tissue. For this, a set of different binary classifiers has been trained and tested. The classifiers showing best performance for this task are identified.
\end{abstract}

Keywords- liver cancer, endogenous fluorescence, minimally invasive interventions, machine learning

\section{INTRODUCTION}

Abdominal cancer is a widely prevalent group of tumours with a high level of mortality if diagnosed at a late stage [1]. Although the cancer death rates have in general declined for decades, the mortality from tumours of the hepatoduodenal area specifically increased in recent years. Moving towards the broader use of minimally invasive techniques for diagnosis and treatment is promising to significantly improve the survival rate and quality of life for patients after the surgery. In this work, we present results of the machine learning approach applied for the optically-guided surgery. We have implemented fluorescent measurements through a flexible fibre optical probe to record the parameters of endogenous fluorescence during minimally invasive interventions in patients with cancers of the hepatoduodenal area [2]. This work aims to develop an appropriate technical implementation for such type of measurements and to assess the efficiency of machine learning methods for real-time diagnosis of tumours in the hepatoduodenal area.

\section{EXPERIMENT AND RESULTS}

The measuring setup consists of a custom-built, flexible fibre optical probe, and two LED sources for the fluoresce excitation (at $365 \mathrm{~nm}$ and $450 \mathrm{~nm}$ ), a set of fluorescent filters, and a CCDbased spectrometer. This prototype of the fibre- optical probe has two emitting fibres to excite the tissue at wavelengths of 365 $\mathrm{nm}$ and $450 \mathrm{~nm}$ and a fibre to collect the fluorescence spectra. The fibre diameters are $400 \mu \mathrm{m}$. This configuration enables to register the fluoresce of all major endogenous fluorophores in living tissue.
The system has been validated in a frame of limited tests in patients with obstructive jaundice caused by pancreatic or liver cancer. The measurements were performed during planned diagnostic and therapeutic interventions under ultrasound and X-ray examination. The studies were conducted at the department of interventional radiology of Orel Regional Clinical Hospital (Orel, Russia) and were approved by the local Committee for Human Biomedical Research Ethics (record of the meeting No10 of 16.11.2017).

To find the most suitable machine learning algorithm for classification of the obtained spectra to come from abnormal or normal tissue, we compared the performance of 5 different algorithms (KNN, a decision tree, SVM, a random forest, and AdaBoost) in terms of their accuracy. This was done separately for every tissue excitation channel. The data preprocessing included filtering the noise, standardisation and principal component analysis of the data. Due to the difficulty of data collection and labelling under realtime surgery operation, we estimate the Bayes error to be high and assume that around $25 \%$ of data is falsely labelled. From this point of view, KNN, the decision tree, and SVM demonstrated the best prediction value with the accuracy achieving the values of $65-71 \%$ on the data. The use of fluorescence spectra registered with the excitation at $450 \mathrm{~nm}$ allows getting better results in comparison with ones obtained with $365 \mathrm{~nm}$ excitation. We are convinced that the proposed technique is highly promising to detect the changes in tissue metabolism for the guided surgery to select surgical tactics. Accordingly, our outlook is to elaborate better data collection and labelling techniques to further improve the overall performance of the system.

\section{ACKNOWLEDGEMENT}

This research was funded by the Russian Science Foundation under grant number 18-15-00201 and MSCA-IF-2017 scheme (ID: 792421).

\section{REFERENCES}

[1] Bray, F.et al. Global cancer statistics 2018: Globocan estimates of incidence and mortality worldwide for 36 cancers in 185 countries. CA: A Cancer J. for Clin. 68, 394-424 (2018).

[2] Kandurova, K. et al. Fiber-optic system for intraoperative study of abdominal organs during minimally invasive surgical interventions. Appl. Sci. 9, 217 (2019) 